\begin{tabular}{|c|l|}
\hline Title & Spin-Filter Device Based on the Rashba Effect Using a Nonmagnetic Resonant Tunneling Diode \\
\hline Author(s) & Koga, Takaaki; Nitta, Junsaku; Takay anagi, Hideaki; Datta, Supriyo \\
\hline Citation & $\begin{array}{l}\text { Physical Review Letters, 88(12), 126601-1-126601-4 } \\
\text { https://doi.org/10.1103/PhysRevLett.88.126601 }\end{array}$ \\
\hline Issue Date & 2002 \\
\hline Doc URL & http://hdl.handle.net/2115/14702 \\
\hline Rights & Copyright $\odot 2002$ A merican Physical Society \\
\hline Type & article \\
\hline File Information & PRL88.pdf \\
\hline
\end{tabular}

Instructions for use 


\title{
Spin-Filter Device Based on the Rashba Effect Using a Nonmagnetic Resonant Tunneling Diode
}

\author{
Takaaki Koga,* Junsaku Nitta, and Hideaki Takayanagi \\ NTT Basic Research Laboratories, NTT Corporation, Atsugi, Kanagawa, 243-0198 Japan
}

Supriyo Datta

School of Electrical and Computer Engineering, Purdue University, West Lafayette, Indiana 47907

(Received 1 October 2001; published 12 March 2002)

\begin{abstract}
We propose an electronic spin-filter device that uses a nonmagnetic triple barrier resonant tunneling diode (TB-RTD). This device combines the spin-split resonant tunneling levels induced by the Rashba spin-orbit interaction and the spin blockade phenomena between two regions separated by the middle barrier in the TB-RTD. Detailed calculations using the InAlAs/InGaAs material system reveal that a splitting of a peak should be observed in the $I-V$ curve of this device as a result of the spin-filtering effect. The filtering efficiency exceeds $99.9 \%$ at the peak positions in the $I-V$ curve.
\end{abstract}

DOI: $10.1103 /$ PhysRevLett.88.126601

Experimental realization of a spin-polarized current source and manipulation of electron spin in a semiconductor are among the most important issues in "spintronics" research [1]. In this research area, extra degrees of freedom provided by electron spins, in addition to those provided by electron charges, are expected to play important roles in realizing new functions in future electronic devices, which include spin-FET [2], spin interference devices [3], and a readout device for the qubit information [4]. In order to explore the roles of spin degrees of freedom in a semiconductor, it is essential to realize a spin-polarized current source from which spin-polarized electrons are injected. The properties of electron spins, including their dynamical motions in the pertinent materials are then studied using the injected spin-polarized electrons. Thus far, various magnetic materials, including ferromagnetic metals [5-7] and diluted magnetic semiconductors [8-13], have been used as spin injection sources. Besides the successes in these approaches, it is also important to develop a spin-polarized current source, or a spin filter, that uses only nonmagnetic semiconductors from the viewpoints of both the attainability of high-quality heterostructures and the absence of the stray magnetic field that may cause some undesirable effects on the spin-filtered electrons.

In this Letter, we propose a spin-filter device that uses a triple barrier (TB) resonant tunneling diode (RTD) which can generate a spin-polarized current without using magnetic properties of materials. Instead, we utilize the Rashba spin-orbit coupling effect [14-16] (Rashba effect) to induce spin-split resonant tunneling levels (RTL) in the proposed device even in the absence of magnetic field. So far, the Rashba effect in a double barrier (DB) RTD has been discussed from both theoretical [17] and experimental points of view [18], and is predicted to provide some degrees of spin polarization in the transmitted electrons [17]. However, the utilization of Rashba effect alone in a DB-RTD does not produce a high degree of spin polarization $(>90 \%)$. To overcome this problem, we propose
PACS numbers: $72.25 . \mathrm{Hg}, 72.25 . \mathrm{Mk}, 73.40 . \mathrm{Ei}, 73.40 . \mathrm{Gk}$

combining the spin-splitting phenomena caused by the Rashba effect with level-matching between the spindependent RTLs, which is accomplished by adjusting the emitter-collector voltage $V_{\mathrm{EC}}$ in the TB-RTD. This level matching procedure is analogous to the recently proposed spin-blockade concept $[4,19]$.

We propose to realize this spin-filter device using a structure illustrated and detailed in Fig. 1(a) and Table I, respectively. A special feature that differentiates the proposed device from other TB-RTDs is the particular mountainlike (triangular) shape in the potential profile as illustrated in Figs. 1(b) and 1(c). This mountainlike potential profile is realized by introducing $n$-type impurities in barriers 1 and 3, and the compensating amount of $p$-type impurities in barrier 2. The actual amount

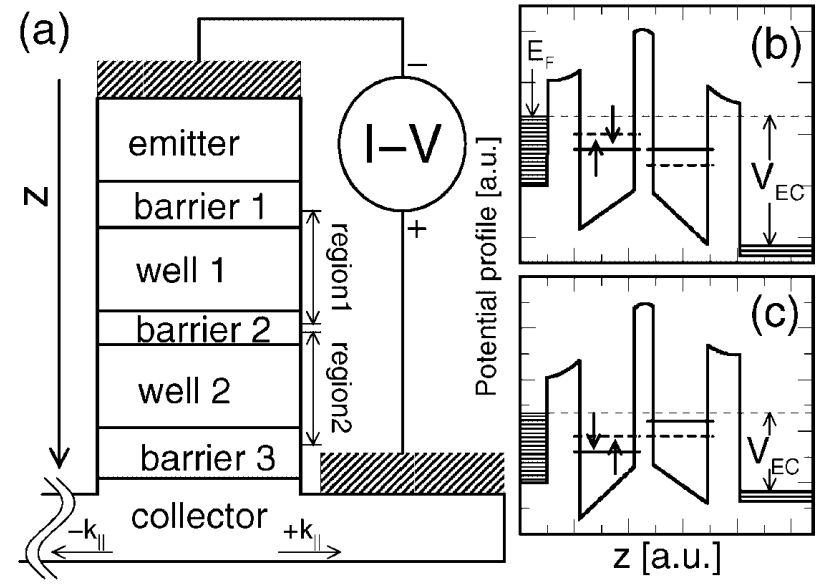

FIG. 1. (a) A schematic illustration of the proposed spin-filter device. The $z$ axis is set vertically, pointing downward. The shaded areas denote the metal electrodes for the $I-V$ measurement. (b),(c) Conduction band potential profiles for the proposed device to show how the matching of spin-dependent resonant tunneling levels is performed by controlling the emittercollector bias voltage $V_{\mathrm{EC}}$. The downward and upward arrows in region 1 denote the clockwise and counterclockwise spin states, respectively. 
TABLE I. Layer information for the proposed spin-filter device.

\begin{tabular}{|c|c|c|c|c|c|c|c|c|c|}
\hline \multirow[b]{2}{*}{ Layer } & \multirow[b]{2}{*}{ Materal } & \multirow[b]{2}{*}{$m^{*} / m_{0}{ }^{\mathrm{a}}$} & \multirow[b]{2}{*}{$E_{g}{ }^{\mathrm{b}}[\mathrm{eV}]$} & \multirow[b]{2}{*}{$\epsilon_{S} / \epsilon_{0}{ }^{\mathrm{c}}$} & \multirow[b]{2}{*}{$\Delta^{\mathrm{d}}$} & \multirow[b]{2}{*}{ Thickness [̊] } & \multicolumn{3}{|c|}{ Impurity densities $^{\mathrm{e}}$} \\
\hline & & & & & & & Structure 1 & Structure 2 & Structure 3 \\
\hline Emitter & $\mathrm{In}_{1-x} \mathrm{Ga}_{x} \mathrm{As}$ & Variable & Variable & N.A. & N.A. & N.A. & $7.74 \times 10^{17}$ & $7.74 \times 10^{17}$ & $7.74 \times 10^{17}$ \\
\hline Barrier 1,3 & $\mathrm{In}_{0.52} \mathrm{Al}_{0.48} \mathrm{As}$ & 0.075 & 1.66 & 12.46 & 0.309 & 60 & $4 \times 10^{18}$ & $2 \times 10^{18}$ & 0 \\
\hline Well 1,2 & $\mathrm{In}_{0.53} \mathrm{Ga}_{0.47} \mathrm{As}$ & 0.041 & 0.783 & 13.1 & 0.328 & 100 & 0 & 0 & 0 \\
\hline Barrier 2 & $\mathrm{In}_{0.52} \mathrm{Al}_{0.48} \mathrm{As}$ & 0.075 & 1.66 & 12.46 & 0.309 & 35 & $1.37 \times 10^{19}$ & $6.85 \times 10^{18}$ & 0 \\
\hline \multirow[t]{2}{*}{ Collector } & $\mathrm{In}_{0.53} \mathrm{Ga}_{0.47} \mathrm{As}$ & 0.041 & 0.783 & 13.1 & 0.328 & N.A. & $\sim 1 \times 10^{17}$ & $\sim 1 \times 10^{17}$ & $\sim 1 \times 10^{17}$ \\
\hline & & & & & & $\begin{array}{l}\Delta E_{c}^{e-b_{1} \mathrm{f}}[\mathrm{eV}] \\
\left|\alpha_{1}\right|^{g}[\mathrm{eVm}]\end{array}$ & $\begin{array}{c}0.270 \\
7.23 \times 10^{12}\end{array}$ & $\begin{array}{c}0.165 \\
3.25 \times 10^{-12}\end{array}$ & $\begin{array}{c}0.030 \\
0\end{array}$ \\
\hline
\end{tabular}

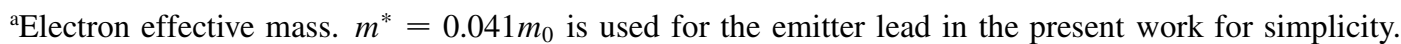

${ }^{\mathrm{b}}$ Energy band gap. $\Delta E_{c} / \Delta E_{g}=0.7$ is used to determine the conduction band offset value.

${ }^{\mathrm{c}, \mathrm{d}}$ Static dielectric constant $\left(\epsilon_{S}\right)$ and spin split-off energy in the valence band $(\Delta)$.

${ }^{\text {e}}$ Barriers 1 and 3 are doped $n$-type, while barrier 2 is doped $p$-type. The emitter and collector leads are doped $n$-type.

${ }^{\mathrm{f}}$ Conduction band offset value between the emitter lead and barrier 1 .

${ }^{g}$ Calculated value for the Rashba constant $\alpha_{1}$ at the middle of region $1 . E=0.04 \mathrm{eV}$ is used for the electron energy.

of these impurities that would cause sufficiently large Rashba spin splittings was determined based on our previous studies of asymmetric quantum wells [16,20]. The $p$ - and $n$-type impurities in the barrier layers are assumed to be completely ionized because the accepter and donor levels in these layers are, respectively, below and above the Fermi levels in the emitter and collector leads. As a result of this particular potential-profile shape, the direction of electric field that an electron feels in region 1 of the device [see Fig. 1(a)] is opposite to that in region 2. Hence, the signs for the corresponding Rashba constants are also opposite each other. In the present work, conduction band offset values between the emitter lead and barrier 1 (denoted as $\Delta E_{c}^{e-b_{1}}$ ) were set smaller than that for the lattice-matched $\mathrm{In}_{0.53} \mathrm{Ga}_{0.47} \mathrm{As} / \mathrm{In}_{0.52} \mathrm{Al}_{0.48} \mathrm{As}$ interface for the purpose of reducing the leakage current in the actual experiment. However, care was taken to make the position of the lowest RTLs still lie higher than that of the conduction band edge of the emitter lead so that electrons can resonantly tunnel through the barriers. Experimentally, the $\Delta E_{c}^{e-b_{1}}$ value can be adjusted by varying the Ga composition $x$ in the $\mathrm{In}_{1-x} \mathrm{Ga}_{x}$ As emitter lead. The value of the Fermi energy in the emitter lead in the present work $\left(E_{\mathrm{F}}=75 \mathrm{meV}\right.$ relative to the conduction band edge) is chosen in such a way that only the lowest RTL can contribute to the total transmission current for all the three proposed device structures listed in Table I.

In order to explain the principle of the proposed device, we consider transmission of an electron with a wave vector $\mathbf{k}$ across the device [21]. In the following explanation, $z$ axis is set perpendicular to the barriers in the RTD. All the energy levels are measured relative to the conduction band edge of the emitter lead. Taking advantage of the circular symmetry of this system about its $z$ axis, we can represent $x$ and $y$ components of the physical quantities by a single "parallel" component (denoted by the subscript II hereafter) without the loss of generality. In the absence of the Rashba effect (or when $k_{\|}=0$ ), an electron with a wave vector $\mathbf{k}$ can transmit through the device if (1) the resonant tunneling level in region 1 (denoted as $E_{r}^{(1)}$ ) matches that in region 2 (denoted as $E_{r}^{(2)}$ ), and (2) the $z$ component of the electron energy in the emitter lead (denoted by $\left.E_{k_{z}} \equiv \frac{\hbar^{2} k_{z}^{2}}{2 m^{*}}\right)$ matches $E_{r}^{(1)}$ and $E_{r}^{(2)}$. While matching between $E_{r}^{(1)}$ and $E_{r}^{(2)}$ is performed by controlling $V_{\mathrm{EC}}$, matching between $E_{k_{z}}$ and $E_{r}^{(1)}$ (or $E_{r}^{(2)}$ ) is automatically made if the resonant levels $E_{r}^{(1)}$ and $E_{r}^{(2)}$ lie between the conduction band edge and the Fermi energy of the emitter lead. Under these conditions, we should observe a single sharp peak in the $I-V$ curve of this device, since condition 1 above can be met only at a certain value of $V_{\mathrm{EC}}$. Now, if the Rashba effect is present and $k_{\|} \neq 0$, the resonant tunneling levels $E_{r}^{(1)}$ and $E_{r}^{(2)}$ experience the level splitting: $E_{r}^{(X)} \rightarrow E_{r}^{(X)} \pm \alpha_{X} k_{\|}(X=1,2)$, where the plus and minus signs correspond to the resonant levels for different spin states, which we denote as "clockwise" $(\downarrow)$ and "counterclockwise" $(\uparrow)$ spins, respectively [22], and $\alpha_{X}$ is the Rashba constant in region $X$. Because of the mirror symmetry in the present device about the middle barrier, the sign for the value of $\alpha_{1}$ is opposite to that of $\alpha_{2}$, while their absolute values are almost equal. Therefore, if the Rashba effect is present and $k_{\|} \neq 0$, the value of $V_{\mathrm{EC}}$ required to match the resonant levels for the clockwise spin $(\downarrow)$ is different from that required for the counterclockwise spin ( $\uparrow$ ) [see Figs. 1(b) and 1(c)]. Hence, we would observe two peaks in the $I$ - $V$ curve.

The values of the actual spin-dependent tunnel current $I_{\uparrow, \downarrow}$ through the proposed device are calculated by integrating the spin-dependent transmission coefficient $T_{L}^{\uparrow, \downarrow}\left(E_{\mathbf{k}_{\|}}, E_{k_{z}}\right)$ across the device with energy $E_{k_{z}}$ and summing the results over all available $\mathbf{k}_{\|}$modes $[23,24]$ :

$$
I_{\uparrow, \downarrow}=\frac{|e|}{h} \sum_{\left|\mathbf{k}_{\|}\right|<k_{\mathrm{F}}} \int_{0}^{E_{\mathrm{F}}-E_{\mathbf{k}_{\|}}} T_{L}^{\uparrow, \downarrow}\left(E_{\mathbf{k}_{\|}}, E_{k_{z}}\right) d E_{k_{z}} .
$$

In Eq. (1), $e$ and $h$ are the electron charge and Planck's constant $(\hbar=h / 2 \pi)$, respectively, and $T=0$ is assumed for temperature, though it turned out that the thermal smearing effect is minimal at least up to $150 \mathrm{~K}$ [25]. It 
is noted that the energies $E_{\mathbf{k}_{\|}}$and $E_{k_{z}}$ are calculated in the emitter lead $\left(E_{\mathbf{k}_{\|}}=\frac{\hbar^{2} k_{\|}^{2}}{2 m^{*}}\right.$ and $\left.E_{k_{z}}=\frac{\hbar^{2} k_{z}^{2}}{2 m^{*}}\right)$, where $m^{*}$ is the electron effective mass. What we are really interested in here is the current injected towards the right $\left(+k_{\|}\right.$ in Fig. 1) in the collector layer which will be detected by our contact and show spin polarization. The current injected towards the left $\left(-k_{\|}\right)$will have exactly the opposite spin polarization since the Rashba field is opposite between $+k_{\|}$and $-k_{\|}$states. However, this current flows away from our contact and will not be detected.

We use the transfer matrix method to calculate $T_{L}^{\uparrow, \downarrow}\left(E_{\mathbf{k}_{\|}}, E_{k_{z}}\right)$, assuming the conservation of $\mathbf{k}_{\|}$across the device $[23,24]$. We consider the following effectively one-dimensional Hamiltonian that describes an electronic state in an in-plane mode $\mathbf{k}_{\|}$with energy $E_{k_{z}}$ :

$$
\frac{\hbar^{2} k_{z}^{2}}{2 m^{*}(z)}+U_{\uparrow, \downarrow}^{\text {eff }}\left(\mathbf{k}_{\|}, z\right)=E_{z},
$$

where $U_{\uparrow, \downarrow}^{\text {eff }}\left(\mathbf{k}_{\|}, z\right)$ is the effective potential energy for clockwise $(\downarrow)$ and counterclockwise $(\uparrow)$ spins, respectively:

$$
U_{\uparrow, \downarrow}^{\text {eff }}\left(\mathbf{k}_{\|}, z\right)=\frac{\hbar^{2} k_{\|}^{2}}{2 m^{*}(z)} \pm \alpha(z) k_{\|}+U_{0}(z)-E_{\mathbf{k}_{\|}} .
$$

In Eq. (3), the second term in the right-hand side corresponds to the energy due to the Rashba effect and the third term corresponds to the conduction band profile in the semiconducting heterostructure. The value of $\alpha(z)$ in Eq. (3) is given by the following equation based on the $\mathbf{k} \cdot \mathbf{p}$-type formalism [15]:

$$
\alpha(z)=\frac{\hbar^{2} E_{p}}{6 m_{0}} \frac{d}{d z}\left(\frac{1}{\Delta E_{\Gamma_{7}}(z)}-\frac{1}{\Delta E_{\Gamma_{8}}(z)}\right),
$$

where $m_{0}$ is the free electron mass, $E_{p}$ is the $\mathbf{k} \cdot \mathbf{p}$ interaction parameter $\left(E_{p}=22 \mathrm{eV}\right.$ is used here [15]), and $\Delta E_{\Gamma_{7}}(z)$ and $\Delta E_{\Gamma_{8}}(z)$ are, respectively, the differences between the electron's total energy $E=E_{\mathbf{k}_{\|}}+E_{k_{z}}$, which is measured from the conduction band edge in the emitter lead, and the energies of the $\Gamma_{7}$ (spin split-off band) and $\Gamma_{8}$ (the highest valence band) valence band edges (denoted as $E_{\Gamma_{7}}$ and $E_{\Gamma_{8}}$, respectively) at position $z$. It is also noted that the difference between $E_{\Gamma_{8}}$ and $E_{\Gamma_{7}}\left(E_{\Gamma_{8}}>E_{\Gamma_{7}}\right)$ is known as the spin split-off energy $\Delta$ in Table I.

The results of the calculation for $I_{\uparrow, \downarrow}$ are shown in Figs. 2(a)-2(c) as a function of $\Delta V_{\mathrm{EC}}\left(\equiv V_{\mathrm{EC}}-V_{\mathrm{EC}}^{0}\right)$ for the three device structures listed in Table I, where, at $V_{\mathrm{EC}}=V_{\mathrm{EC}}^{0}$, the potential profile in region 2 becomes exactly the mirror image of that in region 1 about the center barrier. Shown in the insets to Figs. 2(a)-2(c) are the conduction band profiles for device structures 1,2, and 3 , respectively, at $V_{\mathrm{EC}}=V_{\mathrm{EC}}^{0}$. In Fig. 2(a), we see a clear separation in the $I-V$ curve peak between the two spin states due to the spin-filtering effect. Since the separation of these two peaks is about $13 \mathrm{mV}$, such a splitting of the $I-V$ curve peak should be observable in a real experiment. In Fig. 2(a), we also find that the spin polarization of the transmitted current $\left(\left|I_{\downarrow}-I_{\uparrow}\right| /\left|I_{\downarrow}+I_{\uparrow}\right|\right)$ exceeds $99.9 \%$
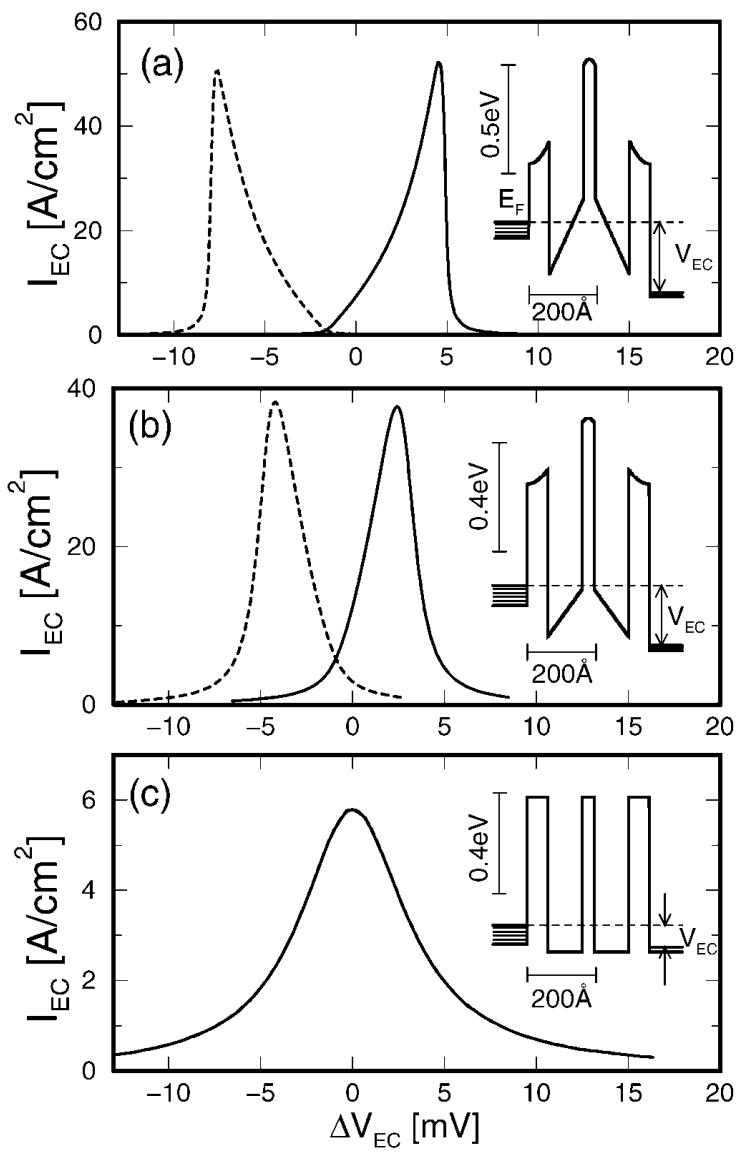

FIG. 2. The calculated spin-dependent tunneling currents as a function of the relative emitter-collector voltage $\Delta V_{\mathrm{EC}} . \Delta V_{\mathrm{EC}}$ is defined as $V_{\mathrm{EC}}-V_{\mathrm{EC}}^{0}$, where the proposed device structures retain a complete mirror symmetry about their middle barriers at $V_{\mathrm{EC}}=V_{\mathrm{EC}}^{0}$. (a), (b), and (c) are the results for device structures 1, 2, and 3 listed in Table I, respectively. Shown in the insets are the conduction band profiles for the corresponding device structures. The dashed and solid curves in the main graphs correspond to the tunnel currents for the clockwise ( $\downarrow$ ) and counterclockwise $(\uparrow)$ spins, respectively. For (c), these two curves overlap.

at the peak positions of the $I-V$ curve, showing the excellent spin-filtering property of the proposed device. A notable feature in Fig. 2(a) is the particular triangularlike asymmetric shape of the $I-V$ curve peak for each spin state. The reason that the $I-V$ curves of the proposed device have this particular shape is the following: As we discussed above, the spin splitting of RTLs for an electron with a wave vector $\mathbf{k}$ is proportional to the value of $k_{\|}$. Therefore, denoting by $V_{\mathrm{EC}}^{\downarrow}$ and $V_{\mathrm{EC}}^{\uparrow}$ the values of $V_{\mathrm{CE}}$ at which the conditions are satisfied for transmitting $\downarrow$ and $\uparrow$ spin electrons with a wave vector $\mathbf{k}$, respectively, we can see that the difference between these voltages $V_{\mathrm{EC}}^{\uparrow}-V_{\mathrm{EC}}^{\downarrow}$ increases as the value of $k_{\|}$increases. In addition, the values of the tunnel current are proportional to the number of $\mathbf{k}_{\|}$modes available for electron transmission. Since the number of these available modes increases proportionally to the value of $k_{\|}$, the value of $I_{\uparrow}$ also increases with increasing $\Delta V_{\mathrm{EC}}$ for $\Delta V_{\mathrm{EC}}>-1.5 \mathrm{meV}$ in Fig. 2(a), for 
example. For $\Delta V_{\mathrm{EC}}>4.5 \mathrm{meV}$ in Fig. 2(a), the value of $I_{\uparrow}$ decreases rapidly to zero. This is because the total energy of an electron with the corresponding $k_{\|}$values $\left(E_{\mathbf{k}_{\|}}+E_{k_{z}}\right)$ exceeds the Fermi energy in the emitter lead in this regime. The behavior of $I_{\downarrow}$ as a function of $\Delta V_{\mathrm{EC}}$ in the corresponding regime is also explained in the same way. Shown in Figs. 2(b) and 2(c) are the results of the calculation for device structures 2 and 3, respectively, where the absolute values of the Rashba constant $|\alpha|$ in regions 1 and 2 are reduced from that in device structure 1 due to the reduced impurity densities in barriers 1,2 , and 3. The results show that the separation of the peaks in the $I-V$ curve reduces as the value of $|\alpha|$ decreases and the splitting of the peak disappears as $|\alpha|$ goes to zero.

A few words about the possible detrimental effects to spin filtering in the proposed device. The charge accumulation in the wells leading to band bending would shift the positions of the RTLs. This effect, if it is so strong that the level matching cannot be made by merely varying the $V_{\mathrm{EC}}$ value, can be circumvented by thickening the emitter-side barrier (reducing the amount of total tunneling current). Ionized impurity scattering in the barrier layers may also lead to the broadening of the RTLs. One can avoid this problem by utilizing composition grading in the wells [26], instead of $p$ and $n$ doping in the barrier layers. This approach would also produce the "mountainlike" potential shape, which is the key configuration for the operation of the proposed device.

In summary, we have proposed a novel spin-filter device that uses a nonmagnetic triple barrier resonant tunneling diode. In this device, the Rashba spin-orbit coupling effect is combined with the spin blockade phenomena to enhance the spin-filtering efficiency, where the values higher than 99.9\% are predicted for the spin-filtering efficiency at the peak positions of the $I-V$ curves. Thus, the excellent spinfiltering properties of this device should play important roles in the future spintronics research.

The authors thank Professor D. B. Janes and Professor P. F. Bagwell at Purdue University and Dr. T. Akazaki at NTT for valuable discussions. This research work was supported by the NEDO International Joint Research Grant Program.

*Also with Nanostructure and Material Property, PRESTO, Japan Science and Technology Corporation (JST). Electronic address: koga@will.brl.ntt.co.jp

[1] G. A. Prinz, Phys. Today 48, No. 4, 58 (1995); Science 282, 1660 (1998).

[2] S. Datta and B. Das, Appl. Phys. Lett. 56, 665 (1990).

[3] T.-Z. Qian and Z.-B. Su, Phys. Rev. Lett. 72, 2311 (1994); J. Nitta, F. E. Meijer, and H. Takayanagi, Appl. Phys. Lett. 75, 695 (1999).

[4] P. Recher, E. V. Sukhorukov, and D. Loss, Phys. Rev. Lett. 85, 1962 (2000).
[5] H. J. Zhu, M. Ramsteiner, H. Kostial, M. Wassermeier, H.-P. Schönherr, and K.H. Ploog, Phys. Rev. Lett. 87, 16601 (2001).

[6] C.-M. Hu, J. Nitta, A. Jensen, J.B. Hansen, and H. Takayanagi, Phys. Rev. B 63, 125333 (2001).

[7] H. B. Heersche, Th. Schäpers, J. Nitta, and H. Takayanagi, Phys. Rev. B 64, 161307 (2001).

[8] J. C. Egues, Phys. Rev. Lett. 80, 4578 (1998).

[9] K. Chang and F. M. Peeters, Solid State Commun. 120, 181 (2001).

[10] Y. Guo, J.-Q. Lu, B.-L. Gu, and Y. Kawazoe, Phys. Rev. B 64, 155312 (2001).

[11] J. C. Egues, C. Gould, G. Richter, and L. W. Molenkamp, Phys. Rev. B 64, 195319 (2001).

[12] R. Fiederling, M. Keim, G. Reuscher, W. Ossau, G. Schmidt, A. Waag, and L. W. Molenkamp, Nature (London) 402, 787 (1999).

[13] Y. Ohno, D. K. Young, B. Beschoten, F. Matsukura, H. Ohno, and D. D. Awschalom, Nature (London) 402, 790 (1999).

[14] E. I. Rashba, Sov. Phys. Solid State 2, 1109 (1960) [Fiz. Tverd. Tela (Leningrad) 2, 1224 (1960)]; Y. A. Bychkov and E. I. Rashba, J. Phys. C 17, 6039 (1984).

[15] G. Engels, J. Lange, T. Schäpers, and H. Lüth, Phys. Rev. B 55, R1958 (1997); T. Schäpers, G. Engels, J. Lange, T. Klocke, M. Hollfelder, and H. Lüth, J. Appl. Phys. 83, 4324 (1998).

[16] J. Nitta, T. Akazaki, H. Takayanagi, and T. Enoki, Phys. Rev. Lett. 78, 1335 (1997).

[17] V. I. Sugarov and Y. A. Yatskevich, Sov. Tech. Phys. Lett. 18, 134 (1992); A. Voskoboynikov, S. S. Liu, and C.P. Lee, Phys. Rev. B 59, 12514 (1999); A. Voskoboynikov, S. S. Lin, C. P. Lee, and O. Tretyak, J. Appl. Phys. 87, 387 (2000).

[18] T. Kikutani, S. Gozu, and S. Yamada, in Proceedings of NGS 10, IPAP Conf. Series 2 (IPAP, Tokyo, 2001), pp. 233; T. Kikutani, S. Gozu, Y. Sato, and S. Yamada, Physica (Amsterdam) E (to be published).

[19] D. Weinmann, W. Häusler, and B. Kramer, Phys. Rev. Lett. 74, 984 (1995).

[20] T. Koga, J. Nitta, T. Akazaki, and H. Takayanagi, in Proceedings of NGS 10 (Ref. [18]), pp. 227; T. Koga, J. Nitta, T. Akazaki, and H. Takayanagi, Physica (Amsterdam) E (to be published).

[21] T. Koga, J. Nitta, T. Akazaki, and H. Takayanagi, Jpn. J. Appl. Phys. (to be published).

[22] The particular spin configuration in a system with the Rashba effect is discussed in Ref. [15]. In short, electron spins are oriented perpendicular to both the in-plane wave vector $\mathbf{k}_{\|}$and the electric field created by the potential profile of the heterostructure.

[23] Y. Ando and T. Itoh, J. Appl. Phys. 61, 1497 (1987).

[24] H. Mizuta and T. Tanoue, The Physics and Application of Resonant Tunnelling Diode (Cambridge University Press, New York, 1995).

[25] T. Koga, J. Nitta, S. Datta, and H. Takayanagi, in Spintronics, edited by T. J. Klemmer, J. Z. Sun, A. Fert, and J. Bass, MRS Symposia Proceedings No. 690 (Materials Research Society, Pittsburgh, to be published).

[26] T. Koga, J. Nitta, T. Akazaki, and H. Takayanagi (unpublished). 